\title{
Coherent States and Symmetric Spaces
}

\author{
A. M. Perelomov \\ Institute for Theoretical and Experimental Physics, Moscow 117259, USSR
}

Received October 15, 1974

\begin{abstract}
Properties of system of the coherent states related to representations of the class I of principal series of the motion groups of symmetric spaces of rank 1 have been studied. It has been proved that such states are given by horospherical kernels and are the generalization of the plane waves for the case of symmetric spaces.
\end{abstract}

\section{Introduction}

This paper deals with the further study of the system of generalized coherent states (CS). According to [1] the system of generalized CS of the type $\left(T,\left|\psi_{0}\right\rangle\right)$ is called the set of states $\left\{T(g)\left|\psi_{0}\right\rangle\right\}$ where $T(g)$ is the unitary irreducible representation (UIR) of the group $G$ acting in the Hilbert space $\mathscr{H}, g$ runs over all the group $G$ and $\left|\psi_{0}\right\rangle$ is the fixed vector of the space $\mathscr{H}$. Such states are a generalization of usual CS $[2,3]$ obtained in choosing the simplest nilpotent group (Heisenberg-Weyl group) as the group $G$ and the so called vacuum vector as $\left|\psi_{0}\right\rangle$. Generalized CS, as well as usual CS, turn out to be very convenient for the solution of a number of physical problems possessing dynamical symmetry.

Thus, for instance, in papers [4] the problems of boson and fermion pair creation in alternating homogeneous field were solved with their help. In paper [5] CS for rotation group (previously introduced in paper [6]) were used to obtain estimates for the partition function of a quantum spin system. In papers $[7,8]$ such states were applied in the so called Dicke model describing the interaction of radiation with the matter.

In the following we shall call generalized CS for brevity simply CS. Note that the CS system is an overcomplete and nonorthogonal system of vectors (states) of Hilbert space. Under the additional assumption on square integrability a number of properties of such systems was considered in papers [9-13].

In this paper we study some CS systems which are not square integrable. namely the systems related to UIRs of the class I of the principal series of symmetric space motion groups. We recall that the representation of class I of the real semisimple Lie group is called the representation $T(g)$ in whose representation space there exists a vector $\left|\psi_{0}\right\rangle$ invariant relative to action of the maximal compact subgroup $K$ of this group. Let us consider the CS system of the type $\left(T,\left|\psi_{0}\right\rangle\right)$. It is easy to see that the coherent state of this type is determined by the point of symmetric space $X=G / K^{1}$. In the case of the so called principal series

${ }^{1}$ In this paper we restrict ourselves by consideration of the case of symmetric spaces of rank I. Note that for the $\mathrm{SO}(3,1)$ group (the Lorenz group) such system of states coincides with that introduced from another considerations in a paper by Shapiro [14]. This system proved to be very convenient for considering a number of problems of the Lorenz group representation theory. For the case of the $\mathrm{SO}(n, 1)$ group a number of useful formulae related to the questions under consideration may be found in book [15]. 
of representations of class I we may use their standard realization [16]. Then the coherent state is determined by the kernel which turns out to be constant on horospheres of symmetric space $X=G / K$. Thereby it is seen the relation of the coherent state method with the horosphere method developed in paper by Gel'fand and Graev [18].

\section{Coherent States Related to Representations of Class I}

Let $G$ be a connected real semisimple Lie group with finite center. It is well known $[16,17]$ that such group possesses a series of representations of class I, i.e. the series of UIRs for which there is the vector $\left|\psi_{0}\right\rangle$ invariant relative to action of maximal compact subgroup $K$ of group $G$. Let us consider the set of states $\left\{T(g)\left|\psi_{0}\right\rangle\right\}$. It can be easily seen that the elements $g_{1}$ and $g_{2}$ which belong to one coset $G$ on $K$ determine one and the same state. Therefore the CS of the type $\left(T,\left|\psi_{0}\right\rangle\right)$ is given by the point of factor-space $X=G / K$. Just choosing some element $g_{x} \in G$ in the coset corresponding to the element $x$ we get the CS system $\{|x\rangle\}$ :

$$
|x\rangle=T\left(g_{x}\right)|0\rangle, \quad|0\rangle=\left|\psi_{0}\right\rangle .
$$

For representations of class I of the so called principal series we may use their explicit realization ${ }^{2}$. It is known (see e.g., [16]) that the group which we consider has the Iwasawa decomposition: $G=K A N$ where $K$ is the maximal compact subgroup of group $G, A$ is Abelian noncompact and $N$ is maximal nilpotent subgroups. Let $M$ be the centralizer of $A$ in $K$, i.e. the set of elements of group $K$ commuting with all elements of group $A$ and $B$ be the subgroup $G$ equal to $M A N$. According to [16] representations of the principal series of class I are called the representations of the group $G$ induced by representations of the subgroup $B$, trivial on $M$.

We recall the construction of induced representation. Let us consider the homogeneous space $\Xi=B \backslash G=M \backslash K$. On this space naturally and transitively act both the group $G$ and its subgroup $K$. Up to a normalization factor there exists a unique $K$-invariant measure $d \mu(\xi)$ which is normalized so that $\int d \mu(\xi)=1$. Let us consider the space of square integrable functions, i.e. those satisfying the condition

$$
\|f\|^{2}=\int|f(\xi)|^{2} d \mu(\xi)<\infty .
$$

Let us choose in each coset corresponding to element $\xi \in \Xi=B \backslash G$ some element $g_{\xi}$. Then arbitrary element of the group $G$ may be written as $g=b g_{\xi}, b \in B$ and the action of the group $G$ on the space $\Xi\left(g: \xi \rightarrow \xi_{g}\right)$ is determined by expansion

$$
g_{\xi} \cdot g=b g_{\eta}, \quad \eta=\xi_{g}, \quad b=\operatorname{man} .
$$

Let us define the action of operator $T(g)$ by the formula

$$
T(g) f(\xi)=\alpha(\xi, g) f\left(\xi_{g}\right)
$$

${ }^{2}$ As is shown in paper [17] the representations of the principal and complementary series of class I for the symmetric space motion groups exhaust all UIRs of class I. 
where $\alpha(\xi, g)$ is the function called multiplier and $\xi_{g}$ is determined from relation (2.3).

It can be easily seen that for the operators $T(g)$ to form representation of the group $G$ it is necessary for the multiplier $\alpha(\xi, g)$ to satisfy functional equation

$$
\alpha\left(\xi, g_{2} g_{1}\right)=\alpha\left(\xi, g_{2}\right) \alpha\left(\xi_{g_{2}}, g_{1}\right) \text {. }
$$

Equation (2.5) is fulfilled if $\alpha(\xi, g)=\left[\frac{d \mu\left(\xi_{g}\right)}{d \mu(\xi)}\right]^{\frac{1}{2}} x(a)$ where $\varkappa(a)$ is the character of the group $A$ and element $a \in A$ is determined by Eq. (2.3). Here if $x(a)$ is the unitary character of the group $A$, then the representation $T(g)$ is unitary and irreducible [17]. Such representation is the representation of class I. This means that a $K$-invariant function exists in the representation space, i.e. a function satisfying functional equation

$$
T(k) f_{0}(\xi)=\alpha(\xi, k) f_{0}\left(\xi_{k}\right)=f_{0}(\xi)
$$

Let us now turn from function $f(\xi)$ to new function $\tilde{f}(\xi)$ according to formula

$$
f(\xi)=f_{0}(\xi) \tilde{f}(\xi) .
$$

Relation (2.4) takes now the form

$$
T(g) \tilde{f}(\xi)=\tilde{\alpha}(\xi, g) \tilde{f}\left(\xi_{g}\right)
$$

where the multiplier $\tilde{\alpha}(\xi, g)$ is given by formula

$$
\tilde{\alpha}(\xi, g)=\frac{f_{0}\left(\xi_{g}\right)}{f_{0}(\xi)} \alpha(\xi, g) \text {. }
$$

It can be easily seen that for all of $k \in K$

$$
\tilde{\alpha}(\xi, k) \equiv 1 \text {. }
$$

Hence it follows $\tilde{\alpha}(\xi, g k)=\tilde{\alpha}(\xi, g)$, i.e. function $\tilde{\alpha}(\xi, g)$ depends only on the coset $x \in X=G \backslash K$ corresponding to element $g$ :

$$
\tilde{\alpha}(\xi, g)=\beta(\xi, x(g)) \text {. }
$$

From relations $\left(2.4^{\prime}\right),(2.9)$ it follows that the function $\tilde{f}_{0}(\xi) \equiv 1$ is invariant relative to transformations of group $K$. Acting on it by the operator $T(g)$ we get the system of coherent states

$$
T(g) \tilde{f_{0}}(\xi)=\tilde{\alpha}(\xi, g)=\beta(\xi, x(g)), \quad x(g)=\pi \cdot g .
$$

Here $\pi: g \rightarrow x(g)$ is a mapping from elements $g$ to corresponding cosets $x$. Thus the coherent state is determined by kernel $\beta(\xi, x)$ where $x \in X$ and $\xi \in \Xi$. It can be shown (see Section 4) that at fixed $\xi$ the function $\beta(\xi, x)$ is constant on horospheres of symmetric space $X=G / K^{3}$, i.e. on the orbits $N_{\xi} \cdot x$ of the nilpotent subgroup $N_{\xi}$ conjugated to $N$ and remaining the point $\xi$ to be fixed. Thus the kernel $\beta(\xi, x)$ may be called horospherical kernel and the coherent state method is in this case

\footnotetext{
3 This statement is valid not only for symmetric spaces of the rank I but also for arbitrary symmetric space.
} 
in close connection with the horosphere method developed in paper by Gel'fand and Graev [18].

Let us consider in more detail the case of symmetric spaces of rank I.

\section{Symmetric Spaces of Rank I}

The rank of symmetric space $G / K$ is called the number of independent metric invariants of a pair of its points. This number equals the dimension of subgroup $A$ of group G. As it is well known (see, e.g. [19]) there exist three series of spaces of rank I and one special space:

I. The real hyperbolic space (Lobachevsky space) of dimension $n$ : $X_{n}^{\mathrm{I}}=S O(n, 1) / S O(n)$ where $S O(n, 1)$ and $S O(n)$ are the groups of real unimodular matrices leaving invariant the form $x_{1}^{2}+\ldots+x_{n}^{2}-x_{n+1}^{2}$ and $x_{1}^{2}+\ldots+x_{n}^{2}$, respectively.

II. The complex hyperbolic space of the real dimension $2 n: X_{n}^{\mathrm{II}}=S U(n, 1)$ / $S U(n) \times U(1)$ where $S U(n, 1)$ and $S U(n)$ are the groups of complex unimodular matrices leaving invariant the forms $\left|z_{1}\right|^{2}+\ldots+\left|z_{n}\right|^{2}-\left|z_{n+1}\right|^{2}$ and $\left|z_{1}\right|^{2}+\ldots+\left|z_{n}\right|^{2}$.

III. The quaternion hyperbolic space of the real dimension $4 n: X_{n}^{\mathrm{III}}=$ $S p(n, 1) / S p(n) \times S p(1)$ where $S p(n, 1)$ and $S p(n)$ are the groups of quaternion unimodular matrices leaving invariant the forms $\left|q_{1}\right|^{2}+\ldots+\left|q_{n}\right|^{2}-\left|q_{n+1}\right|^{2}$ and $\left|q_{1}\right|^{2}+\ldots+\left|q_{n}\right|^{2}$, respectively. Here $|q|$ is the norm of quaternion $q$.

Remind that the quaternion algebra is associative but non-commutative. This is the algebra over the field of real numbers whose basic elements $e_{0}, e_{1}, e_{2}$, and $e_{3}$ satisfy the following multiplication law:

$$
\begin{aligned}
& e_{0}^{2}=e_{0}, \quad e_{i}^{2}=-e_{0}, \quad e_{0} e_{i}=e_{i} e_{0}=e_{i}, \quad i=1,2,3 \\
& e_{1} e_{2}=-e_{2} e_{1}=e_{3}, \quad e_{2} e_{3}=-e_{3} e_{2}=e_{1}, \quad e_{3} e_{1}=-e_{1} e_{3}=e_{2} .
\end{aligned}
$$

Thus, arbitrary quaternion $q$ is of the form of $q=q^{0} e_{0}+q \boldsymbol{e}$ where $\boldsymbol{q}=\left(q^{1}, q^{2}, q^{3}\right)$, $\boldsymbol{e}=\left(e_{1}, e_{2}, e_{3}\right)$.

Let $\bar{q}=q^{0} e_{0}-q \boldsymbol{e}$ be the quaternion conjugated to quaternion $q$. Then the norm of quaternion is $|q|^{2}=\bar{q} q=\left(q^{0}\right)+\boldsymbol{q}^{2}$.

IV. Two-dimensional hyperbolic space over the algebra (non-associative) of the Cayley numbers (octonions) of the real dimension 16:

$X^{\mathrm{IV}}=F_{4}^{R} / S O(9)$ where $F_{4}^{R}$ is the certain real form of special simple group $F_{4}$, $S O(9)$ is the group of orthogonal unimodular matrices of order $9^{4}$.

Note that all three series of symmetric spaces may be realized in a unified way, namely in all three cases one may assume that the group $G=\{g\}$ is a group of matrices $g=\left(\begin{array}{ll}A & B \\ C & D\end{array}\right)$, where $A$ is the $n \times n$ matrix, $B$ is the $n \times 1$ matrix, $C$ is the $1 \times n$ matrix and $D$ is the $1 \times 1$ matrix and the elements of matrices are real numbers, complex numbers or quaternions, respectively. In this case the matrix $g$ must leave invariant the form $\left|x_{1}\right|^{2}+\ldots+\left|x_{n}\right|^{2}-\left|x_{n+1}\right|^{2}$ where $x_{i}$ is the real num-

${ }^{4}$ A number of useful information on the algebra of the Cayley numbers and on geometry of this space may be found in paper [20]. 
ber, complex number or quaternion, respectively. This implies definite conditions on the matrices $A, B, C$, and $D$, in particular

$$
|D|^{2}-\sum_{i=1}^{n}\left|C_{i}\right|^{2}=1, \quad|D|^{2}-\sum_{i=1}^{n}\left|B_{i}\right|^{2}=1 .
$$

Correspondingly, the symmetric space $X=G / K$ in all of three cases may be regarded as the set of vectors $x=\left(x_{1}, \ldots, x_{n}\right)$ satisfying the condition

$$
\left|x_{1}\right|^{2}+\ldots+\left|x_{n}\right|^{2}<1
$$

and the space $\Xi=B \backslash G$ as a set of vectors $\xi=\left(\xi_{1}, \ldots \xi_{n}\right)$ such that

$$
\left|\xi_{1}\right|^{2}+\ldots+\left|\xi_{n}\right|^{2}=1 \text {. }
$$

The group $G$ acts in the space $X$ (correspondingly in the space $\Xi$ ) as the group of "linear fractional transformations"

$$
g: x \rightarrow x^{\prime}=x_{g}, \quad x_{i}^{\prime}=\left(A_{i j} x_{j}+B_{i}\right)\left(C_{j} x_{j}+D\right)^{-1} .
$$

As for the space $X^{\mathrm{IV}}$, it represents the hyperbolic Cayley plane, i.e. it may be considered as a set of vectors $x=\left(x_{1}, x_{2}\right)$ where $x_{1}$ and $x_{2}$ are the Cayley numbers (octonions).

Remind that the octonion algebra is noncommutative and nonassociative but alternative. This means that any two of its elements generate an associative subalgebra. The basis of the Cayley algebra consists of eight elements $e_{0}, e_{1}, \ldots e_{7}$. The norm of the element $x=x^{0} e_{0}+\sum_{i=1}^{7} x^{i} e_{i}$ is given by formula $|x|^{2}=\bar{x} \cdot x=$ $x \cdot \bar{x}=\left(x^{0}\right)^{2}+\sum_{i=1}^{7}\left(x^{i}\right)^{2}$ where $\bar{x}=x^{0} e_{0}-\sum_{i=1}^{7} x^{i} e_{i}$ is the element conjugated to element $x$. The elements of the Cayley algebra may be also represented as pairs of quaternions $x=\left(q_{1}, q_{2}\right)$. In this case the multiplication law is given by formula

$$
\left(q_{1}, q_{2}\right)\left(q_{1}^{\prime}, q_{2}^{\prime}\right)=\left(q_{1} q_{1}^{\prime}-\bar{q}_{2}^{\prime} q_{2}, q_{2}^{\prime} q_{1}+q_{2} \bar{q}_{1}^{\prime}\right)
$$

It may be easily seen that function $\alpha(\xi, g)=\left[\frac{d \mu\left(\xi_{g}\right)}{d \mu(\xi)}\right]^{\frac{1}{2}+i \lambda^{\prime}}$ where $\lambda^{\prime}$ is the real number satisfies the functional equation (2.5) and consequently, is the multiplier for representation of the class I of principal series.

Calculating $\frac{d \mu\left(\xi_{g}\right)}{d \mu(\xi)}$ with the help of Eq. (3.3) we get

$$
\begin{aligned}
& \frac{d \mu\left(\xi_{g}\right)}{d \mu(\xi)}=\left|C_{i} \xi_{i}+D\right|^{-2 \varrho}, \\
& \alpha(\xi, g)=\left|C_{j} \xi_{j}+D\right|^{-\varrho+i \lambda}, \quad \lambda=-2 \varrho \lambda^{\prime}
\end{aligned}
$$

where

$$
\begin{aligned}
& 2 \varrho=\operatorname{dim} X+q^{\prime}-1, \\
& q^{\prime}=\left\{\begin{array}{lll}
0 & \text { for } & X_{n}^{\mathbf{I}} \\
1 & \text { for } & X_{n}^{\mathrm{II}} \\
3 & \text { for } & X_{n}^{\mathrm{II}} \\
7 & \text { for } & X^{\mathrm{IV}}
\end{array}\right.
\end{aligned}
$$


The number $\varrho$ entering formulae (3.5) and (3.6) may be expressed via internal characteristics of symmetric space. Let for this consider the structure of the space $X$ in more detail. It follows from the Iwasawa decomposition that the Lie algebra $\mathscr{G}$ of the group $G$ has the form of $\mathscr{G}=\mathscr{K}+\mathscr{A}+\mathscr{N}$ where $\mathscr{K}, \mathscr{A}$, and $\mathscr{N}$ are the Lie algebras of the subgroups $K, A$, and $N$, respectively. In our case $\mathscr{A}$ is onedimensional: $A=\mathbb{R} H$. It is well known that in the nilpotent subalgebra $\mathscr{N}$ one may choose the basis $X_{\alpha}^{i}$ and $X_{2 \alpha}^{j}$ so that

$$
\begin{aligned}
{\left[H, X_{\alpha}^{i}\right] } & =\alpha X_{\alpha}^{i}, \quad i=1,2, \ldots p \\
{\left[H, X_{2 \alpha}^{j}\right] } & =2 \alpha X_{2 \alpha}^{j}, \quad j=1,2, \ldots q .
\end{aligned}
$$

The elements of algebra $X_{\alpha}^{j}$ form a root subspace $\mathscr{G}_{\alpha}$ corresponding to the $\operatorname{root} \alpha$, respectively $\mathscr{G}_{2 \alpha}=\left\{X_{2 \alpha}^{j}\right\}$. The numbers $p=\operatorname{dim} \mathscr{G}_{\alpha}$ and $q=\operatorname{dim} \mathscr{G}_{2 \alpha}$ are called the multiplicities of roots $\alpha$ and $2 \alpha$. The comparison of (3.8) with the numbers $q$ for spaces $X_{n}^{\mathrm{I}}, X_{n}^{\mathrm{II}}, X_{n}^{\mathrm{III}}$, and $X^{\mathrm{IV}}$ shows that $q^{\prime}=q$. Note more that Eq. (3.7) may be rewritten as

$$
\varrho=\frac{p}{2}+q .
$$

Concluding this section let us give the table of some characteristics of symmetric spaces of rank I

Table 1

\begin{tabular}{llllllllll}
\hline$G$ & $\operatorname{dim} G$ & $K$ & $\operatorname{dim} K$ & $M$ & $\operatorname{dim} M$ & $\operatorname{dim} X$ & $p$ & $q$ \\
\hline$S O(n, 1)$ & $\frac{n(n+1)}{2}$ & $S O(n)$ & $\frac{n(n-1)}{2}$ & $S O(n-1)$ & $\frac{(n-1)(n-2)}{2}$ & $n$ & $n-1$ & 0 & $\frac{n-1}{2}$ \\
$S U(n, 1)$ & $n(n+2)$ & $S U(n) \times U(1)$ & $n^{2}$ & $S U(n-1)$ & $(n-1)^{2}$ & $2 n$ & $2 n-2$ & 1 & $n$ \\
& & & $\times U(1)$ & & & & & \\
$S p(n, 1)$ & $(n+1)(2 n+3)$ & $S p(n) \times S p(1)$ & $n(2 n+1)$ & $S p(n-1)$ & $(n-1)(2 n-1)$ & $4 n$ & $4 n-4$ & 3 & $2 n+1$ \\
$F_{4}^{R}$ & 52 & $S O(9)$ & 36 & $S O(7)$ & 21 & 16 & 8 & 7 & 11 \\
\hline
\end{tabular}

\section{Properties of the Coherent State System}

Acting with the operating $T(g)$ on the function $f_{0}(\xi) \equiv 1$ with the help of (3.6) we get the explicit expression for CS in the $\xi$-representation

$$
\langle\xi, \lambda \mid x\rangle=\Psi_{x}^{\lambda}(\xi)=\left(1-|x|^{2}\right)^{\frac{\varrho-i \lambda}{2}}|1-\bar{x} \cdot \xi|^{-\varrho+i \lambda}
$$

where $\bar{x} \cdot \xi=\sum_{i=1}^{n} \bar{x}_{i} \cdot \xi_{i}$.

In a number of cases another form is more convenient

$$
\Psi_{x}^{\lambda}(\xi)=H_{x}^{\lambda}(\xi)=\left|x_{0}-\check{x} \cdot \bar{\xi}\right|^{-\varrho+i \lambda}
$$

where

$$
x_{0}=\left(1-|x|^{2}\right)^{-1 / 2}, \quad \check{x}=\left(1-|x|^{2}\right)^{-1 / 2} \cdot x .
$$


Thus in this form CS is determined by a point of hyperboloid $\left|x_{0}\right|^{2}-|\check{x}|^{2}=1$ and the function $H_{x}^{\lambda}(\xi)$ is the kernel of integral transform mapping the function $f\left(x_{0}, \check{x}\right)$ on hyperboloid $\left|x_{0}\right|^{2}-|\check{x}|^{2}=1$ to the function on the cone $\left|\xi_{0}\right|^{2}-|\xi|^{2}=0^{5}$.

The CS system is overcomplete and nonorthogonal and possesses a number of remarkable properties which we now consider ${ }^{6}$.

1. The state $|x\rangle$ is normalized to unity

$$
\langle x \mid x\rangle=\left\|\Psi_{x}^{\lambda}\right\|^{2}=\int\left|\Psi_{x}^{\lambda}(\xi)\right|^{2} d \mu(\xi)=1 \text {. }
$$

This follows from the unitarity of representation $T^{2}(g)$.

2. The system $\{|x\rangle\}$ is complete. This follows from irreducibility of $T^{\lambda}(g)$ representation.

3. The operator $T^{\lambda}(g)$ transforms one CS into another

$$
T^{\lambda}(g)|x\rangle=\left|x^{\prime}\right\rangle, \quad x^{\prime}=x_{g-1} .
$$

Equation (4.5) may be easily checked by direct calculation.

4. At fixed $\xi$ function $\Psi_{x}^{\lambda}(\xi)$ is constant on horospheres

$$
\frac{|1-\bar{x} \xi|^{2}}{1-|x|^{2}}=\text { const } \quad \text { or } \quad\left|x_{0}-\check{x} \xi\right|^{2}=\text { const } \text {. }
$$

Thus the kernel $\langle\xi, \lambda \mid x\rangle$ may be called horospherical and the CS method in this case is closely connected with the horosphere method developed in the paper by Gel'fand and Graev [18].

5. Note that the kernel

$$
P(x, \xi)=\left|\Psi_{x}^{\lambda}(\xi)\right|^{2}=\left(\frac{1-|x|^{2}}{|1-\bar{x} \xi|^{2}}\right)^{\varrho}
$$

is the Poisson kernel for symmetric space $X=G / K$. Thus

$$
\Psi_{x}^{\lambda}(\xi)=P(x, \xi)^{\frac{1}{2}+i \lambda^{\prime}} .
$$

6. At fixed $\xi$ function $\Psi_{x}^{\lambda}(\xi)$ is the eigenfunction of the Laplace-Beltrami operator $\Delta_{x}$ for symmetric space $X=\left\{x:|x|^{2}<1\right\}$

$$
-\Delta_{x} \Psi_{x}^{\lambda}(\xi)=\left(\varrho^{2}+\lambda^{2}\right) \Psi_{x}^{\lambda}(\xi) .
$$

Besides, such functions are constants on horospheres which are the analogues of hyperplanes in Euclidean space. Thus the CS are a natural generalization of "plane waves" $\langle\boldsymbol{n}, k \mid \boldsymbol{r}\rangle=e^{i k \boldsymbol{n} \boldsymbol{r}},|\boldsymbol{n}|=1$ for the case of Euclidean space.

7. The CS are nonorthogonal to each other. Their scalar product is given by the formula

$$
\langle x \mid y\rangle=\left\langle 0\left|T^{+}\left(g_{x}\right) T\left(g_{y}\right)\right| 0\right\rangle=\langle 0|T(h)| 0\rangle=\Phi_{\lambda}(\tau)
$$

${ }^{5}$ It should be however taken into account that in cases II and III the action of groups $S U(n, 1)$ and $S p(n, 1)$ on corresponding hyperboloids and cones is not transitive. Let us more note that for $\lambda \rightarrow \infty, \tau \rightarrow 0$, and $\lambda \tau=$ const we get the case of flat space. In this case the coherent states go into plane waves for spaces of the type I-IV, respectively.

6 Note that though a number of properties of the coherent state system having been checked only for symmetric spaces of the type $X_{n}^{\mathrm{I}}, X_{n}^{\mathrm{II}}, X_{n}^{\mathrm{III}}$ all of them seem to remain valid for space $X^{\mathrm{IV}}$ also. 
where

$$
h=g_{x}^{-1} g_{y}=k_{1} a(\tau) k_{2}, \quad \operatorname{ch}^{2} \tau=\frac{|1-\bar{x} y|^{2}}{\left(1-|x|^{2}\right)\left(1-|y|^{2}\right)}
$$

and the $\tau=\tau(x, y)$ determines the distance between points $x$ and $y$ of the space $X$.

From (4.11) it is easy to get the expression for the metrics of symmetric space $X=G / K$ :

$$
\begin{aligned}
d s^{2} & =d \tau^{2}+\operatorname{sh}^{2} \tau\left[d \bar{\xi}_{i} d \xi_{i}-\left(\bar{\xi}_{i} d \xi_{i}\right)\left(d \bar{\xi}_{j} \cdot \xi_{j}\right)\right]+\frac{1}{4} \operatorname{sh}^{2} 2 \tau\left(\bar{\xi}_{i} d \xi_{i}\right)\left(d \bar{\xi}_{j} \xi_{j}\right) \\
x & =\operatorname{th} \tau \cdot \xi, \quad|\xi|^{2}=1 .
\end{aligned}
$$

8. The function

$$
\Phi_{\lambda}(\tau)=\left\langle 0\left|T^{\lambda}(g)\right| 0\right\rangle, \quad g=k_{1} a(\tau) k_{2}
$$

determines the scalar product of two CS. It is called zonal spherical function and plays an important role in the theory of symmetric spaces [19]. It may be also determined by the equation

$$
\Phi_{\lambda}(\tau)=\langle 0 \mid x\rangle=\int \Psi_{x}^{\lambda}(\xi) d \mu(\xi), \quad|x|=\text { th } \tau .
$$

From Eq. (4.14) the integral representations for zonal spherical functions are obtained. For the space $X_{n}^{\mathrm{I}}$

$$
\Phi_{\lambda}(\tau)=\int_{0}^{\pi}[\operatorname{ch} \tau-\operatorname{sh} \tau \cos \theta]^{-\varrho+i \lambda} d \mu(\theta)
$$

where

$$
d \mu(\theta)=\frac{\Gamma\left(\frac{p+1}{2}\right)}{\sqrt{\pi} \Gamma\left(\frac{p}{2}\right)}(\sin \theta)^{p-1} d \theta
$$

In other cases

$$
\Phi_{\lambda}(\tau)=\int_{0}^{\frac{\pi}{2}} \int_{0}^{\pi}\left[(\operatorname{ch} \tau-\operatorname{sh} \tau \cos \theta \cos \varphi)^{2}+\operatorname{sh}^{2} \tau \cos ^{2} \theta \sin ^{2} \varphi\right]^{\frac{-\varrho+i \lambda}{2}} d \mu(\theta, \varphi)
$$

where

$$
d \mu(\theta, \varphi)=\frac{2 \Gamma\left(\frac{p+q+1}{2}\right)}{\sqrt{\pi} \Gamma\left(\frac{p}{2}\right) \Gamma\left(\frac{q}{2}\right)}(\sin \theta)^{p-1}(\cos \theta)^{q}(\sin \varphi)^{q-1} d \theta d \varphi .
$$

These functions are even $\Phi_{\lambda}(-\tau)=\Phi_{\lambda}(\tau)$ and are normalized by a condition $\Phi_{\lambda}(0)=1$, besides $\Phi_{-\lambda}(\tau)=\Phi_{\lambda}(\tau)$.

9. The zonal spherical function $\Phi_{\lambda}(\tau)$ is an eigenfunction of the radial part of the Laplace-Beltrami operator for the symmetric space

$$
-\left(\frac{d^{2}}{d \tau^{2}}+p \operatorname{cth} \tau \frac{d}{d \tau}+2 q \operatorname{cth} 2 \tau \frac{d}{d \tau}\right) \Phi_{\lambda}(\tau)=\left(\varrho^{2}+\lambda^{2}\right) \Phi_{\lambda}(\tau) .
$$


Solving this equation we get the expression for zonal spherical function in terms of hypergeometric function [21]

$$
\Phi_{\lambda}(\tau)=F\left(a, b, c ;-\operatorname{sh}^{2} \tau\right)
$$

where

$$
a=\frac{\varrho+i \lambda}{2}, \quad b=\frac{\varrho-i \lambda}{2}, \quad c=\frac{p+q+1}{2} .
$$

10. The Laplace-Beltrami operator is a selfadjoint operator. Hence it follows that zonal spherical functions satisfy the orthogonality relation (at $\lambda>0$ and $\lambda^{\prime}>0$ )

$$
\begin{aligned}
& \int_{0}^{\infty} \bar{\Phi}_{\lambda}(\tau) \Phi_{\lambda^{\prime}}(\tau) d \mu(\tau)=N(\lambda) \delta\left(\lambda-\lambda^{\prime}\right), \\
& d \mu(\tau)=(2 \operatorname{sh} \tau)^{p}(2 \operatorname{sh} 2 \tau)^{q} d \tau .
\end{aligned}
$$

Hence it also follows that $\int_{0}^{\infty}\left|\Phi_{\lambda}(\tau)\right|^{2} d \mu(\tau)=\infty$, i.e. the considered CS system is not square-integrable.

11. Here the normalizing coefficient $N(\lambda)$ is determined by the asymptotics of function $\Phi_{\lambda}(\tau)$ at $\tau \rightarrow \infty$

$$
\Phi_{\lambda}(\tau) \sim\left(c(\lambda) e^{i \lambda \tau}+c(-\lambda) e^{-i \lambda \tau}\right) e^{-\varrho \tau}, \quad \bar{c}(\lambda)=c(-\lambda), \quad \tau \rightarrow \infty .
$$

From Eqs. (4.20), (4.21) we get

$$
N(\lambda)=2 \pi|c(\lambda)|^{2}
$$

and from explicit expression (4.18) for $\Phi_{\lambda}(\tau)$ we get $^{7}$

$$
\begin{aligned}
c(\lambda) & =2^{\varrho-i \lambda} \Gamma\left(\frac{p+q+1}{2}\right) \frac{\Gamma(i \lambda)}{\Gamma\left(\frac{\varrho+i \lambda}{2}\right) \Gamma\left(\frac{p}{4}+\frac{1}{2}+\frac{i \lambda}{2}\right)}, \\
N(\lambda) & =2^{p+2 q+1} \pi\left|\Gamma\left(\frac{p+q+1}{2}\right)\right|^{2} \frac{|\Gamma(i \lambda)|^{2}}{\left|\Gamma\left(\frac{p}{4}+\frac{1}{2}+\frac{i \lambda}{2}\right)\right|^{2}\left|\Gamma\left(\frac{\varrho+i \lambda}{2}\right)\right|^{2}} .
\end{aligned}
$$

12. Let us consider the space of functions $f(\tau)$ satisfying the condition

$\int_{0}^{\infty}|f(\tau)|^{2} d \mu(\tau)<\infty$.

The functions $\Phi_{\lambda}(\tau)$ form the complete system in this space, and the completeness condition has the form

$$
\int_{0}^{\infty} \bar{\Phi}_{\lambda}(\tau) \Phi_{\lambda}\left(\tau^{\prime}\right) d \mu(\lambda)=\frac{1}{(2 \operatorname{sh} \tau)^{p}(2 \operatorname{sh} 2 \tau)^{q}} \delta\left(\tau-\tau^{\prime}\right), \quad d \mu(\lambda)=\frac{d \lambda}{2 \pi|c(\lambda)|^{2}} .
$$

13. The relations (4.20) and (4.26) enable one to expand arbitrary function satisfying condition (4.25) in zonal spherical functions:

$$
f(\tau)=\int \hat{f}(\lambda) \Phi_{\lambda}(\tau) d \mu(\lambda)
$$

7 The formula (4.23) for the function $c(\lambda)$ is a particular case of the general formula by Gindikin and Karpelevič [22]. 
where the coefficient $\hat{f}(\lambda)$ is determined by the formula

$$
\hat{f}(\lambda)=\int \bar{\Phi}_{\lambda}(\tau) f(\tau) d \mu(\tau) .
$$

14. Here the Plancherel formula is valid

$$
\int_{0}^{\infty}|f(\tau)|^{2} d \mu(\tau)=\int_{0}^{\infty}|\hat{f}(\lambda)|^{2} d \mu(\lambda)
$$

15. Analogous relations of orthogonality are valid for the functions describing the coherent states. Namely

$$
\int \bar{\Psi}_{x}^{\lambda}(\xi) \Psi_{x}^{\lambda^{\prime}}\left(\xi^{\prime}\right) d \mu(x)=N(\lambda) \delta\left(\lambda-\lambda^{\prime}\right) \delta\left(\xi, \xi^{\prime}\right)
$$

where

$$
x=\operatorname{th} \tau \cdot \hat{x}, \quad|\hat{x}|=1, \quad d \mu(x)=d \mu(\tau) d \mu(\hat{x}), \quad \int d \mu(\hat{x})=1 .
$$

Besides

$$
\int \bar{\Psi}_{x}^{\lambda}(\xi) \Psi_{x^{\prime}}^{\lambda}(\xi) d \mu(\xi) d \mu(\lambda)=\delta\left(x, x^{\prime}\right) .
$$

Here delta-functions $\delta\left(\xi, \xi^{\prime}\right)$ and $\delta\left(x, x^{\prime}\right)$ are different from zero only for $\xi^{\prime}=\xi$ and $x^{\prime}=x$ and satisfy the relations

$$
\int \delta\left(\xi, \xi^{\prime}\right) d \mu\left(\xi^{\prime}\right)=1, \quad \int \delta\left(x, x^{\prime}\right) d \mu\left(x^{\prime}\right)=1 .
$$

Relation (4.32) follows immediately from identity

$$
\int \bar{\Psi}_{x}^{\lambda}(\xi) \Psi_{x^{\prime}}^{\lambda}(\xi) \mathrm{d} \mu(\xi)=\Phi_{\lambda}\left(\tau\left(x, x^{\prime}\right)\right)
$$

and from the condition of completeness of zonal spherical functions (4.26).

Relations of completeness (4.30) follow from the Schur lemma. Let us remark that after integration relations (4.30) on $d \mu\left(\xi^{\prime}\right)$ we get the relations of orthogonality (4.20).

16. Relations (4.30) and (4.32) allow one to expand an arbitrary function $f(x)$ satisfying the condition

$$
\int|f(x)|^{2} d \mu(x)<\infty
$$

in the coherent states

$$
\begin{aligned}
& f(x)=\int \hat{f}(\xi, \lambda) \Psi_{x}^{\lambda}(\xi) d \mu(\xi) d \mu(\lambda), \\
& \hat{f}(\xi, \lambda)=\int \bar{\Psi}_{x}^{\lambda}(\xi) f(x) d \mu(x)
\end{aligned}
$$

and the Plancherel formula holds

$$
\int|f(x)|^{2} d \mu(x)=\int|\hat{f}(\xi, \lambda)|^{2} d \mu(\xi) d \mu(\lambda) .
$$

17. The zonal spherical functions are closely connected with quantummechanical scattering problem in the certain potential $V(\tau)$. To see this fact substitute into Eq. (4.17) new function

$$
\tilde{\Phi}_{\lambda}(\tau)=(2 \operatorname{sh} \tau)^{p / 2}(2 \operatorname{sh} 2 \tau)^{q / 2} \Phi_{\lambda}(\tau) .
$$


For the function $\tilde{\Phi}_{\lambda}(\tau)$ we get the equation which has the form of the Schrödinger equation with potential $V(\tau)$

$$
\left(-\frac{d^{2}}{d \tau^{2}}+V(\tau)\right) \tilde{\Phi}_{\lambda}(\tau)=\lambda^{2} \tilde{\Phi}_{\lambda}(\tau)
$$

where

$$
\begin{aligned}
V(\tau) & =\frac{a}{\operatorname{sh}^{2} \tau}+\frac{b}{\operatorname{sh}^{2} 2 \tau}, \\
a & =\left[\left(\frac{p+q-1}{2}\right)^{2}-\left(\frac{q-1}{2}\right)^{2}\right], \quad b=\left[(q-1)^{2}-1\right] .
\end{aligned}
$$

The result is that asymptotic behaviour at $\tau \rightarrow \infty$ the function $\tilde{\Phi}_{\lambda}(\tau)$ has the form

$$
\tilde{\Phi}_{\lambda}(\tau) \sim\left(c(\lambda) e^{i \lambda \tau}+c(-\lambda) e^{-i \lambda \tau}\right) .
$$

18. The scattering in the potential $V(\tau)$ is determined by the so called scattering matrix (see, for example, book [23]) which in this case has the form

$$
S(\lambda)=-c(\lambda) / c(-\lambda) \text {. }
$$

Using the expression (4.23) for $c(\lambda)$ we get

$$
S(\lambda)=-2^{-2 i \lambda} \frac{\Gamma(i \lambda) \Gamma\left(\frac{\varrho-i \lambda}{2}\right) \Gamma\left(\frac{p}{4}+\frac{1}{2}-\frac{i \lambda}{2}\right)}{\Gamma(-i \lambda) \Gamma\left(\frac{\varrho+i \lambda}{2}\right) \Gamma\left(\frac{p}{4}+\frac{1}{2}+\frac{i \lambda}{2}\right)} .
$$

Let us remark that functions $c(\lambda)$ and $S(\lambda)$ are meromorphic functions and that function $c(\lambda)$ have no zeroes (and no poles) in the lower semiplane $\lambda$, and this fact corresponds to the absence of bound states (discrete spectrum) in this problem. In the upper semiplane the function $c(\lambda)$ [and correspondingly the function $S(\lambda)$ ] has zeroes and poles on imaginary semiaxis $\lambda=i x, x>0$. These poles are related to the fact that potential $V(\tau)$ decreases at $\tau \rightarrow \infty$ too slowly (only exponentially) and no bound states correspond to them. As for zeroes of the function $c(\lambda)$ in the upper semiplane in all cases except for $S O(n, 1)$ group for odd $n$ they correspond to bound states (discrete spectrum) in the problem of symmetric space of the compact type which is dual according to Cartan to the space $X$.

Representation $T^{2}(g)$ being restricted on the maximal compact subgroup $K$ is decomposed into irreducible representations of this group.

Let $\hat{K}$ be the set of all UIRs of the group $K$, and $\hat{K}_{0}$ be the set of representations entering in decomposition of representation $T^{\lambda}(g)$. It is known that any representation which belong to $\hat{K}_{0}$ enters in this decomposition only once. From the reciprosity theorem by Frobenius [16] it follows that $\hat{K}_{0}$ contains only those representations of the group $K$ which being restricted on the subgroup $M$ contains the identity representation.

It is seen from Table 1 that the problem is reduced to consideration of restriction of groups $S O(n) \rightarrow S O(n-1), S U(n) \rightarrow S U(n-1), S p(n) \rightarrow S p(n-1)$ and 
$S O(9) \rightarrow S O(7)$, respectively. Remind that the representation of the compact simple group of rank $v$ is determined by integer nonnegative numbers $l_{1}, \ldots l_{v}$. Making use of the result given in the book [24] we get the following answer:

$$
\begin{array}{ll}
\text { In case I } & \hat{K}_{0}=\{(l, 0, \ldots 0)\} \\
\text { In case II } & \hat{K}_{0}=\left\{\left(l_{1}, 0, \ldots 0, l_{2}\right)\right\} \\
\text { In case III } & \hat{K}_{0}=\left\{\left(l_{1}, l_{2}, 0, \ldots 0\right)\right\} \\
\text { In case IV } & \hat{K}_{0}=\left\{\left(l_{1}, l_{2}, 0,0\right)\right\} .
\end{array}
$$

20. Coherent state $|x\rangle$ may be expanded in representations entering $\hat{K}_{0}$. Denoting the basis functions of representation $D_{l}$ entering $\hat{K}_{0}$ through $|l, m\rangle$, $\langle\xi \mid l m\rangle=Y_{l m}(\xi)$ where $l$ is the set of numbers characterising the representation and $m$ is the set of numbers enumerating functions transforming by the representation $D_{l}$ we get the analog of expansion of the "plane wave"

$$
\begin{aligned}
& |x\rangle=\sum_{l m} \Phi_{\lambda l m}(x)|l m\rangle, \\
& \Psi_{x}^{\lambda}(\xi)=\sum \Phi_{\lambda l m}(x) Y_{l m}(\xi), \\
& \Phi_{\lambda l m}(x)=\langle l m|T(g)| 0\rangle .
\end{aligned}
$$

From normalization condition for $|x\rangle$ it follows that

$$
\sum_{l m}\left|\Phi_{\lambda l m}(x)\right|^{2}=1 \text {. }
$$

21. Note that Eq. (4.48) is equivalent to integral representation for the function

$$
\Phi_{\lambda l m}(x)=\int \bar{Y}_{l m}(\xi) \Psi_{x}^{\lambda}(\xi) d \mu(\xi) .
$$

It may be also shown that

$$
\Phi_{\lambda l m}(x)=\Phi_{\lambda l}(\tau) \bar{Y}_{l m}(\hat{x}), \quad x=\text { th } \tau \cdot \hat{x}, \quad \Phi_{-\lambda l}(\tau)=\Phi_{\lambda l}(\tau) .
$$

Expansion (4.47) takes now the form

$$
\Psi_{x}^{\lambda}(\xi)=\sum_{l m} \Phi_{\lambda l}(\tau) \bar{Y}_{l m}(\hat{x}) Y_{l m}(\xi)=\sum_{l m} \Phi_{\lambda l}(\tau) \sqrt{d_{l}} Y_{l 0}(|\hat{x} \bar{\xi}|)
$$

where $x=\operatorname{th} \tau \cdot \hat{x}$, and $d_{l}$ is the dimension of the representation $D_{l}$ of the group $K$. The explicit expression for $d_{l}$ is given by the well known formulae by Weyl.

22. The functions $\Phi_{\lambda l m}(x)$ being the matrix elements of the operator $T^{\lambda}(g)$ are the eigenfunctions of the Laplace-Beltrami operator $\Delta_{x}$ on symmetric space

$$
\begin{aligned}
& -\Delta_{x} \Phi_{\lambda l m}(x)=\left(\varrho^{2}+\lambda^{2}\right) \Phi_{\lambda l m}(x), \\
& \Delta_{x}=\left(1-|x|^{2}\right)\left(\frac{\partial^{2}}{\partial x_{i} \partial \bar{x}_{i}}-x_{i} \bar{x}_{j} \frac{\partial}{\partial x_{i}} \frac{\partial}{\partial \bar{x}_{j}}\right) .
\end{aligned}
$$

23. The functions $\Phi_{\lambda l}(\tau)$ (the so called associated spherical functions) are orthogonal to each other

$$
\int \bar{\Phi}_{\lambda l}(\tau) \Phi_{\lambda^{\prime} l}(\tau) d \mu(\tau)=N_{l}(\lambda) \delta\left(\lambda-\lambda^{\prime}\right) .
$$


24. Coefficient $N_{l}(\lambda)$ is determined by the asymptotic of the function $\Phi_{\lambda l}(\tau)$ at $\tau \rightarrow \infty$

$$
\Phi_{\lambda l}(\tau) \sim\left(c_{l}(\lambda) e^{i \lambda \tau}+c_{l}(-\lambda) e^{-i \lambda \tau}\right) e^{-\varrho \tau}
$$

here $c_{l}(\lambda)=\sqrt{d_{l}} c(\lambda), N_{l}(\lambda)=2 \pi\left|c_{l}(\lambda)\right|^{2}$ where $d_{l}$ is the dimension of the representation $D_{l}$.

25. The functions $\Phi_{\lambda l}(\tau)$ form complete system. The condition of completeness in this case has the form

$$
\int \bar{\Phi}_{\lambda l}(\tau) \Phi_{\lambda l}\left(\tau^{\prime}\right) d \mu_{l}(\lambda)=\frac{1}{(2 \operatorname{sh} \tau)^{p}(2 \operatorname{sh} 2 \tau)^{q}} \delta\left(\tau-\tau^{\prime}\right), \quad d \mu_{l}(\lambda)=\frac{d \lambda}{2 \pi|c(\lambda)|^{2} \cdot d_{l}} .
$$

26. Analogous to the case of zonal spherical functions we can expand arbitrary function $f(\tau)$ such that

$$
\int_{0}^{\infty}|f(\tau)|^{2} d \mu(\tau)<\infty
$$

in associated spherical functions $\Phi_{\lambda l}(\tau)$ :

$$
f(\tau)=\int \hat{f}_{l}(\lambda) \Phi_{\lambda l}(\tau) d \mu_{l}(\lambda)
$$

where

$$
\hat{f}_{l}(\lambda)=\int \bar{\Phi}_{\lambda l}(\tau) f(\tau) d \mu(\tau)
$$

and

$$
\int|f(\tau)|^{2} d \mu(\tau)=\int\left|\hat{f}_{l}(\lambda)\right|^{2} d \mu_{l}(\lambda)
$$

27. Note that the function

$$
\tilde{\Phi}_{\lambda l}(\tau)=(2 \operatorname{sh} \tau)^{p / 2}(2 \operatorname{sh} 2 \tau)^{q / 2} \Phi_{\lambda l}(\tau)
$$

satisfies equation of the type of Schrödinger equation with potential

$$
\begin{aligned}
V_{l}(\tau) & =\frac{a_{l}}{\operatorname{sh}^{2} \tau}+\frac{b_{l}}{\operatorname{sh}^{2} 2 \tau}, \\
a_{l} & =\left[\left(l_{1}+\frac{p+q-1}{2}\right)^{2}-\left(l_{2}+\frac{q-1}{2}\right)^{2}\right], \quad b_{l}=4\left[\left(l_{2}+\frac{q-1}{2}\right)^{2}-\frac{1}{4}\right] .
\end{aligned}
$$

Here coefficients $a_{l}$ and $b_{l}$ are eigenvalues of certain operators $-\Delta_{1}$ and $-\Delta_{2}$ acting on the sphere $|x|=$ const.

Hence it follows that the asymptotics of the function $\Phi_{\lambda l}(\tau)$ has the form of (4.55) and the functions $\Phi_{\lambda l}(\tau)$ and $c_{l}(\lambda)$ are obtained from the functions $\Phi_{\lambda}(\tau)$ and $c(\lambda)$ by replacing

$$
p \rightarrow p+2\left(l_{1}-l_{2}\right), \quad q \rightarrow q+2 l_{2} .
$$

Let us note in conclusion that the results analogous to those of the present paper may be also obtained for the case of symmetric spaces of arbitrary rank. This problem is considered in a paper [25].

Acknowledgements. Thanks are due to M. Baker who read the manuscript of the paper and helped to eliminate a number of miscorrections in translating into English. 


\section{References}

1. Perelomov, A. M.: Commun. math. Phys. 26, 222 (1972)

2. Glauber, R. J.: Phys. Rev. 130, 2529 (1963); 131, 2766 (1963)

3. Klauder, J.R., Sudarshan E.C.G.: Fundamentals of quantum optics, New York: Benjamin 1968

4. Perelomov, A. M.: Phys. Lett. A 39, 165, 353 (1972); Teor. Mat. Fiz. 16, 303 (1973); 19, 83 (1974)

5. Lieb,E.H.: Commun. math. Phys. 31, 327 (1973)

6. Radcliffe, J. M.: J. Phys. A 4, 313 (1971)

7. Arecchi,F.T., Courtens, E., Gilmore, R., Thomas, H.: Phys. Rev. A 6, 2211 (1972)

8. Hepp, K., Lieb, E.H., Phys. Rev. A 8, 2517 (1973)

9. Perelomov, A. M.: Teor. Mat. Fiz. 6, 213 (1971)

10. Bargmann, V., Butera, P., Girardello,L., Klauder, J. R.: Reps. Math. Phys. 2, 221 (1971)

11. Perelomov, A. M.: Funct. Anal. Appl. 6, No 4, 47 (1972)

12. Perelomov, A. M.: Funct. Anal. Appl. 7, No 3, 57 (1973)

13. Monastyrsky, M.I., Perelomov,A. M.: Dokl. Akad. Nauk SSSR 207, 1303 (1972); Reps. Math. Phys. 6, 1 (1974)

14. Shapiro, I. S.: Dokl. Akad. Nauk SSSR 106, 647 (1956)

15. Vilenkin, N. Y.: Special functions and the theory of group representations. Providence, R. I.: Amer. Math. Soc. 1968

16. Mackey, G. W.: Bull. Amer. Math. Soc. 69, 628 (1963)

17. Kostant, B.: Bull. Amer. Math. Soc. 75, 627 (1969)

18. Gel'fand, I. M., Graev, M.I.: Trudy Moskov. Math. Obsč. 8, 321 (1959)

19. Helgason, S.: Differential geometry and symmetric spaces. New York: Academic Press, 1962

20. Freudenthal, H.: Oktaven, Ausnahmengruppen und Oktavengeometrie, Utrecht, 1951

21. Harish-Chandra: Amer. J. Math. 80, 241 (1958)

22. Gindikin, S. G., Karpelevič, F. I.: Dokl. Akad. Nauk SSSR 145, 252 (1962)

23. Landau, L.D., Lifshitz,E.M.: Quantum mechanics. London: Pergamon Press, 1958

24. Zhelobenko,D.P.: Compact Lie groups and their representations. Moscow: Nauka, 1970

25. Monastyrsky, M.I., Perelomov, A. M.: Ann. Inst. H. Poincaré, sec. A. 23, 23 (1975)

Communicated by H. Araki 